University of Texas at El Paso

ScholarWorks@UTEP

$1-2019$

\title{
High Concentrations Naturally Lead to Fuzzy-Type Interactions and to Gravitational Wave Bursts
}

Oscar Galindo

The University of Texas at El Paso, ogalindomo@miners.utep.edu

Olga Kosheleva

The University of Texas at El Paso, olgak@utep.edu

Vladik Kreinovich

The University of Texas at El Paso, vladik@utep.edu

Follow this and additional works at: https://scholarworks.utep.edu/cs_techrep

Part of the Computer Sciences Commons

Comments:

Technical Report: UTEP-CS-19-02

\section{Recommended Citation}

Galindo, Oscar; Kosheleva, Olga; and Kreinovich, Vladik, "High Concentrations Naturally Lead to FuzzyType Interactions and to Gravitational Wave Bursts" (2019). Departmental Technical Reports (CS). 1292. https://scholarworks.utep.edu/cs_techrep/1292

This Article is brought to you for free and open access by the Computer Science at ScholarWorks@UTEP. It has been accepted for inclusion in Departmental Technical Reports (CS) by an authorized administrator of ScholarWorks@UTEP. For more information, please contact Iweber@utep.edu. 


\title{
High Concentrations Naturally Lead to Fuzzy-Type Interactions and to Gravitational Wave Bursts
}

\author{
Oscar Galindo, Olga Kosheleva, and Vladik Kreinovich \\ University of Texas at El Paso \\ 500 W. University \\ El Paso, Texas 79968, USA \\ ogalindomo@miners.utep.edu, olgak@utep.edu, \\ vladik@utep.edu
}

\begin{abstract}
Fuzzy logic is normally used to describe the uncertainty of human knowledge and human reasoning. Physical phenomena are usually described by probabilistic models. In this paper, we show that in extremal conditions, when the concentrations are very large, some formulas describing physical interactions become fuzzy-type. We also show the observable consequences of such fuzzy-type formulas: they lead to bursts of gravitational waves.
\end{abstract}

\section{Traditional Viewpoint: Probabilities for Physical World, Fuzzy for Imprecise ("Fuzzy") Expert Knowledge}

Equations describing the physical world are usually precise. Traditionally, our knowledge is described in precise terms. From Newton's laws to relativity theory, we have precise equations that describe how different physical quantities change in time.

Sometimes, this knowledge is probabilistic. In many physical situations, it is not possible to exactly predict the future state of a system.

In some situations, we know the exact equations describing the interaction of particles, but the number of particles is so huge that it is not possible to exactly solve the corresponding system of equations. For example, a room full of air contains about $10^{23}$ molecules. In this case, all we can do is make predictions about the frequency of different outcomes - i.e., about the probabilities of different events. This is the case of statistical physics; see, e.g., [2, 12]. 
If we take quantum effects into account, then the same phenomenon occurs for all possible physical processes. Indeed, according to quantum physics, it is not even theoretically possible to predict the exact future values of all the physical quantities such as coordinates, momentum, etc. [2, 12]. All we can do is predict the corresponding probabilities.

Physical equations describing the probabilities are also exact. While the knowledge is probabilistic, equations that describe how these probabilities change with time are exact, be it Boltzmann's equations of statistical physics or Schroedinger's equations of quantum mechanics.

These equations are usually smooth (differentiable). Different particles are reasonable independent, so the overall probability can be obtained by multiplying probabilities corresponding to different particles. The product function is differentiable infinitely many times, so usually, the corresponding equations are also differentiable (smooth).

Need to describe expert knowledge. In many real-life situations, in addition to (or, sometimes, instead of) the exact equations, we also have imprecise ("fuzzy") expert knowledge, knowledge that experts describe by using imprecise natural-language words.

For example, a medical doctor may say that a skin irritation is suspicious if it has irregular shape, or that a medicine is recommended if the patient has a high fever - but what exactly is irregular or high is not well-defined, it is subjective.

Fuzzy logic as a natural way to describe imprecise expert knowledge. In both above examples, it is not the case that we have an exact threshold on temperature (or on some regularity parameter) so that:

- below this threshold, we have one decision, and

- above the threshold, we have another decision.

This would make no sense: why give a medicine to someone whose body temperature is $39.00 \mathrm{C}$ but but not to someone whose temperature is $38.99 \mathrm{C}$ ?

In all such cases, for temperatures close to some transition value, experts are not $100 \%$ sure whether the temperature is high (or whether the shape is irregular), they are only confident to some degree.

In the computer, "absolutely true" is usually represented as 1, and "absolutely false" as 0 , so it is reasonable to describe intermediate degrees of confidence by intermediate numbers, i.e., numbers from the interval $[0,1]$. This is the main behind fuzzy logic, a formalism invented by Lotfi A. Zadeh to describe imprecise expert knowledge; see, e.g., [1, 3, 7, 9, 10, 13].

In fuzzy logic, to describe each imprecise property like "high", we assign, to each possible value $q$ of the corresponding quantity, a number $\mu(q)$ from the interval $[0,1]$ that describes to what extent the expert is confident that this quantity satisfies the corresponding property - e.g., to what extent the given temperature $q$ is high. 
How do we estimate fuzzy degrees. To estimate the expert's degrees of confidence, we can, e.g., ask each expert to mark his/her degree of confidence on a scale from 0 to 10 :

- 0 meaning no confidence at all, and

- 10 meaning absolutely sure.

To get a value between 0 and 1 , we divide the resulting estimate by 10 .

Need to combine fuzzy degrees. Experts can estimate degrees of certainty in their statements. However, conclusions based on expert knowledge are often based on taking into account several expert statements. Our degree of confidence in such a conclusion is thus equal to our degree of confidence that the first of used statements is true and the second used statement is true, etc. In other words, in addition to the expert's degrees of confidence in their statements $S_{1}, \ldots, S_{n}$, we also need to estimate the degrees of confidence in different "and"-combinations $S_{i} \& S_{j}, S_{i} \& S_{j} \& S_{k}$, etc.

In the ideal world, we can simply ask the experts to estimate the degree of confidence in each such combination. However, this is not realistically possible. Indeed, for $n$ original statements, there are $2^{n}-1$ such combinations: since combinations are in 1-1 correspondence with non-empty subsets of the set of $n$ statements. Already for reasonable $n=30$, we get an astronomical number $2^{30} \approx 10^{9}$ combinations - and there is no way that we can ask a billion questions to the experts.

Since we cannot elicit the expert's degree of confidence in "and"combinations directly from the experts, we therefore need to estimate these degrees based on the known experts' degrees of confidence in individual statements. In other words, we need to be able to combine the degrees of confidence $a$ and $b$ of statements $A$ and $B$ into an estimate for degree of confidence in the "and"-combination $A \& B$. The algorithm for such combination is called an "and"-operation or, for historical reasons, a t-norm. The result of applying this combination algorithm to numbers $a$ and $b$ will be denoted $f_{\&}(a, b)$.

How to combine fuzzy degrees? Which operation $f_{\&}(a, b)$ should we choose?

First, since $A \& B$ means the same as $B \& A$, it is reasonable to require that the resulting estimates coincide as well, i.e., that $f_{\&}(a, b)=f_{\&}(b, a)$.

Second, since $A \& A$ means the same as $A$, it is reasonable to require that our estimate for the degree of confidence in $A \& A$ be the same as the original degree of confidence in the statement $A$, i.e., that $f_{\&}(a, a)=a$.

Third, since $A \& B$ is a stronger statement than each of $A$ and $B$ - since it implies both $A$ and $B$-it is reasonable to require that our degree of confidence in this "and"-statement is smaller than or equal to the degree of confidence in each $A$ and $B: f_{\&}(a, b) \leq a$ and $f_{\&}(a, b) \leq b$.

Finally, if our degree of confidence in one or both of the statements $A$ and $B$ increases, the resulting degree of confidence in $A \& B$ should also increase or at least remain the same (but definitely not decrease). In other words, the 
function $f_{\&}(a, b)$ should be monotonic: if $a \leq a^{\prime}$ and $b \leq b^{\prime}$, then we should have $f_{\&}(a, b) \leq f_{\&}\left(a^{\prime}, b^{\prime}\right)$.

It turns out that there is exactly one operation that satisfies these four properties: the min-operation $f_{\&}(a, b)=\min (a, b)$ (and this operation, proposed in the very first of Zadeh's papers, is indeed one of the most widely used ones in applications of fuzzy techniques). Indeed, it is easy to see that the min-operation satisfies all four above properties.

Vice versa, let us assume that a function $f_{\&}(a, b)$ satisfies all four properties. Since this function is symmetric (first property), it is sufficient to consider the case when $a \leq b$. In this case, due to the third property, we have

$$
f_{\&}(a, b) \leq a .
$$

On the other hand, since $a \leq b$, monotonicity implies that $f_{\&}(a, a) \leq f_{\&}(a, b)$. By the second property, $f_{\&}(a, a)=a$, so we get

$$
a \leq f_{\&}(a, b) .
$$

From $f_{\&}(a, b) \leq a$ and $a \leq f_{\&}(a, b)$, we conclude that $f_{\&}(a, b)=a$ for $a \leq b$, i.e., that for $a \leq b$, we have $f_{\&}(a, b)=\min (a, b)$. Due to symmetry, this equality holds for $a \geq b$ as well. The statement is proven.

\section{High Concentrations Naturally Lead to Fuzzy-Type Interactions}

Chemical kinetics and Boltzmann's formulas of statistical physics: a brief reminder. As we have mentioned earlier, in many real-life situations, we have a large number of small interacting particles. These particles may be molecules of different type whose interaction constitute chemical reactions, these particles may be molecules of gas whose interaction simply means bouncing off each other, etc.

The molecules interact when they are close to each other. The usual way to describe such an interaction takes into account that, on the microscopic level, the space is mostly empty, so such interactions are reasonably rare. As a result, e.g., in chemical reactions, the probability that a molecule of the 1st type gets involved in the interaction in a given time period is proportional to the probability of finding any molecule of the 2nd type, and this probability is, in its turn, proportional to the concentration $b$ of the molecules of the 2 nd type. To get the overall amount of interactions, we need to multiply this probability by the number of molecules of the 2st type - which is proportional to their concentration $a$. Thus, the intensity of intersection is proportional to the product $a \cdot b$ of the two concentrations.

The rate with which each of the concentration changes is therefore also proportional to this product:

$$
\frac{d a}{d t}=k \cdot a \cdot b+\ldots
$$


When the reaction leads to physical motion, the corresponding change in the location $x$ is also proportional to the product:

$$
\frac{d x}{d t}=k \cdot a \cdot b+\ldots
$$

Beyond traditional models: case of high concentration. What happens when the concentration is high? This situation is best understood if we consider yet another phenomenon where the above product model is usually applied - the predator-prey model. For example, if both wolves and rabbits are reasonably rare, it takes some running for a wolf to find a rabbit, and the intensity of the wolves-eat-rabbits process is proportional to the product $a \cdot b$ of the concentrations of wolves and rabbits.

But what if the concentrations of wolves and rabbits become so large that they can, in effect, always see each other? In this case, each wolf is actively engaged in eating rabbits - as long as there is sufficient number of rabbits for each of the wolves. Thus:

- If the concentration of wolves $a$ is smaller than the concentration of rabbits $b$, the intensity of the eating process is proportional to the number of wolves, i.e., to $a$.

- On the other hand, if there are fewer rabbits than wolves, i.e., if $a>b$, the the intensity of eating is proportional to the number of rabbits, i.e., to $b$.

Both cases can be described by a single formula: namely, in both cases, the intensity of interaction - and thus, the rate with which each concentration changes - is proportional to $\min (a, b)$ (see, e.g., $[4,5,6]$ ):

$$
\begin{aligned}
& \frac{d a}{d t}=k \cdot \min (a, b)+\ldots \\
& \frac{d x}{d t}=k \cdot \min (a, b)+\ldots
\end{aligned}
$$

Discussion. This model is indeed fuzzy-type:

- in contrast to the traditional product formula which is similar to the usual formula for the probability of the combined event $A \& B$ (see, e.g., [11]),

- the new formula resembles a widely used formula for finding the fuzzy degree of confidence of such a combined event.

Comment. It is worth mentioning that fuzzy-type interactions are not only useful for describing high-concentration physical phenomena. Since these interactions correspond to faster reaction, their simulation can speed up computations $[4,5,6]$. 


\section{Fuzzy-Type Interactions Naturally Lead to Gravitational Wave Bursts}

Can we detect remote fuzzy-type interactions? When we are in the vicinity of high-concentration processes - e.g., in catalysis - we can directly observe the fuzzy-type behavior. However, most physical processes with high concentrations are in the domain of astrophysics, thousands of light years away from us. Is it possible to distinguish such faraway fuzzy-type processes from more regular one?

The main difference between fuzzy-type and traditional interactions. Traditional interactions are smooth. In a small vicinity of each location, each smooth function can be well approximated by linear functions - just like a curve can be approximated by its tangent. This possibility to linearize a smooth function - i.e., to replace it by linear terms in its Taylor expansion in small vicinity of each location - is an important idea that helps physicists in their computations; see, e.g., [2]. From this viewpoint, in a small vicinity, all smooth interactions are similar - they are all linear.

Interestingly, this is where fuzzy-type interactions differ:

- while the right-hand side of the formula (1a) - corresponding to traditional interactions - is always smooth (differentiable),

- the right-hand side of the formula (2a) - corresponding to fuzzy-type interactions - is not differentiable in the vicinity of the line $a=b$.

To be more precise, the corresponding derivatives are discontinuous at this point: for the function $f(a, b)=\min (a, b)$ :

- we have $\frac{\partial f}{\partial a}=1$ when $a<b$ and thus, $f(a, b)=a$, and

- we have $\frac{\partial f}{\partial a}=0$ when $a>b$ and thus, $f(a, b)=b$.

We cannot use this difference directly. Can we use this difference in smoothness to directly distinguish non-smooth fuzzy-type interactions from smooth traditional ones? Not really: even when we observe an Earth object from far away, the image is blurred (i.e., in mathematical terms, smoothed). In general, all remote signals are smoothed, so, irrespective of whether the original signal was smooth or not, the observed signal is smooth.

Let us try to observe the difference indirectly. While we cannot observe the non-smoothness directly, we may be able to observe it indirectly. Good news is that in physics, the rate of change - i.e., the derivative - of a quantity is also an observable quantity.

For example, we can observe coordinates - and measure the corresponding location of an object. We can also directly measure the first derivative of the coordinate - the velocity - e.g., by its Doppler effect. We can even directly 
measure the second derivative of the coordinate - the acceleration - by an accelerometer.

In our case, the first derivative $\frac{d x}{d t}$ is described by a non-smooth function $\min (a, b)$. Thus, the derivative of the first derivative - i.e., the acceleration $\frac{d^{2} x}{d t^{2}}$ - is discontinuous. Discontinuity, however, will also be smoothed.

Let us go one step further and take one more derivative, i.e., let us consider the third derivative $\frac{d^{3} x}{d t^{3}}$. For a "jump" function, the derivative is infinite, so we will have an infinite third derivative. After smoothing, we will still have a huge value in the vicinity of the original non-smoothness. So, the question is: how can observe the third derivative?

How can we observe the third derivative: analysis of the problem. In most real-life measurements, the first two derivatives dominate - to the extent that, in contrast to the the first two derivatives that correspond to named physical quantities (velocity and acceleration), there is no special word for the third derivative. To be able to observe the third derivative, we thus need to come up with a physical phenomenon in which the first two derivatives do not dominate - i.e., when their effects are 0s (or at least small).

In other words, we need a physical phenomenon in which accelerations do not count: if we have several bodies moving with the same acceleration, it is as if they are immobile. There is a well-known phenomenon of this type: namely, this is exactly what General Relativity and gravity are all about [2, 8, 12]. Indeed, Einstein's discovery of General Relativity started with his Equivalence Principle, according to which a person in a freely falling elevator will not notice any gravitation.

In General Relativity, there is no absolute space or absolute time, what we measure when we measure spatial distances or time intervals depends on the bodies. In particular, if in some coordinate system, all the bodies move with the same acceleration $\vec{a}$, this simply means that we can change the coordinate system by taking the location of one of the bodies as the system's starting point. In the new coordinate system, all the bodies have 0 acceleration relative to each other - thus, there is no acceleration at all.

The situation is different is we have a change in acceleration - third derivative - be it change in time or change across space. This change cannot be eliminated by simply changing the coordinate system. Thus, our hope for detecting third derivatives lies in the analysis of the gravitation phenomena.

How can we find the observable consequences of third derivative in gravitation? Gravitation force is very weak in comparison to all other physical forces. As a result, many phenomena are very difficult to observe and measure for gravity - since they are very weak.

Good news is that - at least on the Newtonian level - the formulas for gravitation are similar to the formulas for the electromagnetic field: in both cases, we have the same Coulomb's law (the only difference is that similar electric charges repulse each other, while similar masses attract each other). 
Another good news is that electromagnetic forces are much stronger than the gravitational force. This can be seen, e.g., by the fact that even small magnets easily overcome gravitation and pick up bodies from the ground, not to mention that electrons forming an electric current are not deterred by gravity and easily climb the wire going up. As a result of this difference in strength, many phenomena which are difficult to observe and measure for gravity (since they are very weak) can be easily observed and measured for electromagnetic processes.

From this viewpoint, to find the observable consequences of third derivative in gravitation, let us recall observable effects of other derivatives in electromagnetic phenomena.

Electromagnetic interactions: a brief reminder. According to Coulomb's laws, all electric changes interact with each other: opposite charges attract each other, while charges of different signs repel each other.

Starting from Newton, interaction between the two bodies was understood as action-at-a-distance, so that a change in one object immediately affects all other objects in the Universe. In this description, interactions travel instantaneously - i.e., with infinite speed,

However, according to relativity theory, no signal can propagate faster than the speed of light; no action-at-a-distance is possible. The only way a particle can affect another particle is via a field: a particle changes the field in its vicinity, this change leads to a change in the vicinity of this vicinity, etc., until the change reaches the location of the second particle.

In quantum physics, everything is quantized, including the fields. Quanta of electromagnetic field are photons. Thus, interaction between two charged particles means, in effect, that one particle emits photons, and photons then interact with other particles. In other words, particles exchange photons - and this exchange results in attraction or repulsion.

In particular, different particles forming a changed body interact with each other by exchanging photons.

When the body is inertial, i.e., when the body travels with a constant speed along a straight line, this process of exchanging photons is stable: some photons go in, some come out, everything is stable, no photons are lost, and no energy is lost. Indeed, otherwise, the loss of energy would mean that the body starts slowing down - and in the coordinate system associated with its initial motion, this would mean that the initially immobile body starts moving, which contradicts to energy conservation law.

However, when a body deviates from the inertial trajectory - i.e., if there is an acceleration - the balance between incoming and outgoing photos is disrupted: the number of photons going on corresponds to one velocity, while the number of photons coming back in corresponds to a different velocity. As a result of this dis-balance, when a body accelerates, some photons are lost and some energy is lost. So, accelerated body emits some photons - i.e., radiates, emits what is called electromagnetic waves.

The larger the deviation from the inertial movement - i.e., the larger the 
acceleration - the larger the resulting photon flow. In the first approximation, the intensity of this photon flow (radiation) is proportional to the acceleration.

Back to gravitational interactions: fuzzy-type interactions lead to gravitational waves. Gravity is also a field, its quanta are called gravitons. From this viewpoint, gravitational interaction means that the bodies exchange gravitons.

In contrast to the electromagnetic field, acceleration does not necessary means disbalance: as we have mentioned, a constant acceleration does not have any physical meaning, it can be easily eliminated by changing a coordinate system. The only thing that cannot be eliminated by such a change is the third derivative. Thus, if there is a third derivative, there is a disbalance between outgoing and incoming gravitons - i.e., a body emits a gravitational wave.

In the first approximate, the intensity of this wave is proportional to the third derivative - i.e., to the intensity of the fuzzy-type interactions.

What type of gravitational waves will be observe? In the fuzzy-type interaction, the locations that have an infinite third derivative - and thus, emit gravitational waves - corresponds to the case when the high concentrations are equal $a(t)=b(t)$.

For a homogeneous body, in general, we have only one moment of time when this equality occurs, so we will see a momentary burst of gravitational waves.

For a non-homogeneous body, at different locations, the above equality is, in general, achieved at different times. So, we will have an extended burst and by the duration of this burst, we can tell how non-homogeneous is the corresponding celestial body.

Comment. It should be mentioned that the expected bursts are different from the gravitational waves that we are observing now [12]: these waves are chirps, periodic waves with a rapidly increasing frequency. A natural explanation for such chirps is that they are caused by two bodies (e.g., two black holes) orbiting closely around each other. As they emit gravitational waves, they lose energy and thus, get closer and closer to each other. This, in accordance with the Kepler's laws, causes the orbiting period to decrease - and thus, the frequency of the emitted waves to increase.

\section{Acknowledgments}

This work was partially supported by the US National Science Foundation via grant HRD-1242122 (Cyber-ShARE Center of Excellence).

\section{References}

[1] R. Belohlavek, J. W. Dauben, and G. J. Klir, Fuzzy Logic and Mathematics: A Historical Perspective, Oxford University Press, New York, 2017. 
[2] R. Feynman, R. Leighton, and M. Sands, The Feynman Lectures on Physics, Addison Wesley, Boston, Massachusetts, 2005.

[3] G. Klir and B. Yuan, Fuzzy Sets and Fuzzy Logic, Prentice Hall, Upper Saddle River, New Jersey, 1995.

[4] O. Kosheleva, V. Kreinovich, and L. Carvalho Barros, "Chemical kinetics in situations intermediate between usual and high concentrations: fuzzy-motivated derivation of the formulas", In: L. Zadeh, R. R. Yager, S. N. Shahbazova, M. Reformat, and V. Kreinovich (eds.), Recent Developments and New Direction in Soft Computing: Foundations and Applications, Springer Verlag, Cham, Switzerland, 2018, pp. 525-536.

[5] V. Kreinovich, "S. Maslov's iterative method: 15 years later (freedom of choice, neural networks, numerical optimization, uncertainty reasoning, and chemical computing)", In: V. Kreinovich and G. Mints (eds.), Problems of Reducing the Exhaustive Search, American Mathematical Society, Providence, Rhode Island, 1997, pp. 175-189.

[6] V. Kreinovich and O. Fuentes, "High-concentration chemical computing techniques for solving hard-to-solve problems, and their relation to numerical optimization, neural computing, reasoning under uncertainty, and freedom of choice", In: E. Katz (ed.), Molecular and Supramolecular Information Processing: From Molecular Switches to Logical Systems, Wiley-VCH, Wienheim, Germany, 2012, pp. 209-235.

[7] J. M. Mendel, Uncertain Rule-Based Fuzzy Systems: Introduction and New Directions, Springer, Cham, Switzerland, 2017.

[8] C. W. Misner, K. S. Thorne, and J. A. Wheeler, Gravitation, Freeman Publ., San Francisco, California, 1973.

[9] H. T. Nguyen, C. Walker, and E. A. Walker, A First Course in Fuzzy Logic, Chapman and Hall/CRC, Boca Raton, Florida, 2019.

[10] V. Novák, I. Perfilieva, and J. Močkoř, Mathematical Principles of Fuzzy Logic, Kluwer, Boston, Dordrecht, 1999.

[11] D. J. Sheskin, Handbook of Parametric and Nonparametric Statistical Procedures, Chapman and Hall/CRC, Boca Raton, Florida, 2011.

[12] K. S. Thorne and R. D. Blandford, Modern Classical Physics: Optics, Fluids, Plasmas, Elasticity, Relativity, and Statistical Physics, Princeton University Press, Princeton, New Jersey, 2017.

[13] L. A. Zadeh, "Fuzzy sets", Information and Control, 1965, Vol. 8, pp. 338353 . 